\section{The cosmic surprise}

Vital Harmonies: Molecular
Biology and our Shared
Humanity

It seems inevitable today that scientists have a difficult time explaining their results to the public. People would like clear-cut answers about the efficacy of a particular drug or the role of diet in cancer, for example, but scientists seem to constantly change their minds and speak in uncertain terms. Scientists also seem to have trouble convincing the public about larger issues - witness the ongoing debates on evolution, global warming, and stem cell research. Often, there is public skepticism as to whether the highly specialized knowledge derived from laboratory science can be meaningfully brought to bear on important issues facing society.

In Vital Harmonies: Molecular Biology and Our Shared Humanity, Erwin Fleissner, a molecular virologist and former dean of sciences and mathematics at the City University of New York, asks: what can molecular biology tell us about our place in the world? Fleissner approaches this question by tracing his own career in biology at a time of explosive advances in molecular biology. Along the way he examines how molecular biology, chemistry and physics have contributed to major ideas concerning biological organisation, brain structure and consciousness, evolutionary biology and ecology. That he is able to accomplish this in a lucid manner while at the same time evoking a sense of wonder at the complexity and beauty of nature is both impressive and moving.

The book starts from a rather unusual perspective: as Fleissner awaits surgery related to a brain tumour, he looks out of the window of his hospital room to see the building in which he carried out cancer research for twenty years and muses on his own research, and on the dramatic growth of molecular biology during his lifetime. His book is a kind of paean to genetics and molecular biology, to those who developed it and to what it has revealed to us about life itself. In the face of the second law of thermodynamics, that dictates ever increasing disorder, he sees the development of living organisms as an unpredictable "cosmic surprise" in which genes direct the channelling of solarderived energy into replication, biological organization and, ultimately, language and culture. That all life on earth is linked via genes in the

Robert N. Eisenman is in the Division of Basic Sciences, Fred Hutchinson Cancer Research Centre, P.O. Box 19024, Seattle, Washington

98109-1024, USA.

e-mail:eisenman@fhcrc.org form of DNA (or RNA), and that our genes record the evolutionary history of our species, are among the surprises reveled, and continually proven anew to us, by genetics and molecular biology have provided us, with. The advent of these fields has resulted in at least one welcome casualty - the notion of "protoplasm" as a kind of amorphous vital substance of the cell has been replaced by ordered assemblies of specific molecules making up distinct structures. Here Fleissner borrows the quantum concept (in which energy exists as discrete units) from physics to describe the discrete nature of the structural, functional, informational and genetic units, in the form of nucleic acids and proteins, that comprise a living cell. He considers cellular behaviour and embryonic development as a result of higher order interactions between these quantal entities to produce a coordinated system with emergent properties that could not be deduced from studying its individual components - hence the need for a 'systems biology. The demise of protoplasm is important as Fleissner wants to do away with vitalism and mystical thinking and, quoting Einstein, argues that we can find both beauty and mystery enough by contemplating “...the marvelous structure of the existing world....

\section{Fleissner is unafraid to draw on physics, philosophy, history, theology and art to animate his arguments.}

However Fleissner is not satisfied with simply describing how molecular biology has revised our thinking about biological problems. In the book's final chapters he makes bold and exhilarating leaps into the nature of consciousness and into broader questions that touch on how evolution can both reveal our connection to nature and serve as an ethical guide. Fleissner is unafraid to draw on physics, philosophy, history, theology and art to animate his arguments. At one point he makes a fascinating analogy between complementarity in atomic physics (for example, the wave/particle duality of the electron) and the complementary ways that the entropic principle and natural selection can been understood to act together to drive evolution.

This slim volume is not, and does not purport to be a molecular biology primer. It will work best for students who are already familiar with the fundamental concepts of genetics and molecular biology and would like to understand their ramifications on a broader context. For scientists, the book will encourage a shift in focus from the minutiae of technical problems and grant writing, perhaps reminding us of what brought many of us into biology in the first place - the idea that studying even a small and simple bit of nature can illuminate the basic principles of all life. This book makes a convincing case for pushing our own ideas to their limits. 